\title{
Blood Flow Velocity Waveforms in the Fetal Cardiac Outflow Tract as a Measure of Fetal Well-Being in Intrauterine Growth Retardation
}

\author{
I. A. L. GROENENBERG, T. STIJNEN, AND J. W. WLADIMIROFF \\ Department of Obstetrics and Gynaecology [I.A.L.G., J.W.W.] and Department of Biostatistics [T.S.], Erasmus \\ University Rotterdam, Rotterdam, The Netherlands
}

\begin{abstract}
Maximum flow velocity waveforms were recorded in a longitudinal study from the fetal ascending aorta and fetal pulmonary artery in $\mathbf{4 6}$ normal pregnancies and, in addition, from the umbilical artery in 21 cases of intrauterine growth retardation between 19 and 33 wk gestation. In normal pregnancy, the mean peak systolic velocity (PSV) in the ascending aorta increased from 49.4 $\mathrm{cm} / \mathrm{s}$ at $19 \mathrm{wk}$ of gestation to $79.0 \mathrm{~cm} / \mathrm{s}$ at $33 \mathrm{wk}$ of gestation. The corresponding increase in PSV in the pulmonary artery was from 39.0 to $63.7 \mathrm{~cm} / \mathrm{s}$. The ratio for the PSV between the two arteries remained constant (1.251.29). Mean values of PSV in both arteries were linearly related to gestational age. Normal limits according to age were constructed by establishing the 5 th and 95 th percentiles. In intrauterine growth retardation, the PSV in the pulmonary artery was decreased (<5th percentile) in $95 \%$ of cases, PSV in the ascending aorta was reduced $(<5$ th percentile) in only $57 \%$. No relationship was established between PSV in both arteries and the presence or absence of end-diastolic flow velocities in the umbilical artery. The outcome of fetuses with intrauterine growth retardation, as expressed by Apgar score at $1 \mathrm{~min}$ and umbilical cord $\mathrm{pH}$, bears no relationship to the PSV in ascending aorta and pulmonary artery. (Pediatr Res 27: 379-382, 1990)
\end{abstract}

\section{Abbreviations}

AO, ascending aorta

IUGR, intrauterine growth retardation

PA, pulmonary artery

PSV, peak systolic velocity

SDS, SD score

Combined two-dimensional real-time imaging and Doppler facilities give reproducible noninvasive information of fetal cardiac function (1-3). Flow velocity waveforms have been studied under both normal and pathologic circumstances, in combination with atrioventricular or outflow tract dimensions for calculation of ventricular stroke volume or output (4-7). However, these calculations are subject to a considerable amount of error due to inaccurate dimension measurements. This is particularly so for smaller fetuses.

The objective of the present study was 3-fold: 1 ) to establish the distribution of peak systolic velocities in the fetal cardiac

Received June 25, 1989; accepted November 24, 1989.

Correspondence: J. W. Wladimiroff, M.D., Ph.D., Professor of Obstetrics and Gynaecology, Academic Hospital Rotterdam-Dijkzigt, Erasmus University Rotterdam, Dr. Molewaterplein 40,3015 GD Rotterdam, The Netherlands.

Supported by Dutch Foundation for Medical Research MEDIGON Grant 900 516-105. outflow tract (ascending aorta and pulmonary artery) during the second half of normal pregnancy; 2) to determine the degree of abnormality of peak systolic velocities in the fetal cardiac outflow tract and its relation to end-diastolic flow velocity in the umbilical artery and birth wt in the presence of IUGR due to impaired placental perfusion; 3) to relate peak systolic velocities in the fetal cardiac outflow tract to fetal well-being as expressed by Apgar score at $1 \mathrm{~min}$ and umbilical arterial $\mathrm{pH}$.

\section{MATERIALS AND METHODS}

A total of 46 normal pregnancies and 21 cases of IUGR consented to participate in the study. The study protocol was approved by the Hospital Ethics Committee. Doppler examinations in 46 normal pregnancies were carried out at 3- to 4-wk intervals between 19 and $33 \mathrm{wk}$ gestation. It is in this particular period of pregnancy that the vast majority of IUGR cases is referred to our department.

Normal pregnancy was defined by a normal fetal biparietal diameter and birth wt between the 5 th and 95 th percentile according to Kloosterman's tables, corrected for maternal parity and fetal sex (8). The pregnancy duration was determined from the last menstrual period and confirmed by ultrasonic measurements of the biparietal diameter between 14 and 18 wk of gestation. The median maternal age was 27 y (range 19-42 y), the median parity was 0 (range $0-3$ ).

IUGR was defined by: 1) flattening of the fetal growth pattern resulting in a clinical discrepancy of more than $2 \mathrm{wk}$ on fundal ht on two successive appointments, combined with an ultrasound finding of the upper abdominal circumference measurement below the 5 th percentile in association with a normal or reduced head circumference measurement (9) and 2) postnatal confirmation by birth wt below the 5th percentile for gestational age, corrected for maternal parity and fetal sex (8). In 14 of 21 cases of IUGR there was pregnancy-induced hypertension. The lag time between flow measurement and delivery in the 21 cases of IUGR was always less than $14 \mathrm{~d}$ (median $3 \mathrm{~d}$ ). If more than one measurement was made per patient, the measurement nearest to the date of delivery was taken for analysis. Median maternal age was 28 y (range 24-37 y), median parity $0(0-3)$. In 17 subjects, flow velocity waveforms from both ascending aorta and pulmonary artery were related to the Apgar score at $1 \mathrm{~min}$; in 15 subjects also to the umbilical artery $\mathrm{pH}$.

A combined mechanical sector and pulsed Doppler system (Diasonics CV 400, Diasonics Inc., Mulpikas, CA) with a carrier frequency of 3.5 and $3.0 \mathrm{MHz}$ was used for blood flow velocity measurements in the ascending aorta, pulmonary artery and umbilical artery. The sector scanner operates at power outputs less than $100 \mathrm{~mW} / \mathrm{cm}^{2}$ spatial peak/temporal average in both imaging and Doppler modes by manufacturers' specifications. Two-dimensional real-time imaging was used to ensure the correct position of the pulsed Doppler gate both before and after 
each Doppler tracing was obtained. Maximum flow velocity waveforms from the ascending aorta were recorded from the "five chamber" view (Fig. 1). Maximum flow velocity waveforms from the pulmonary artery were collected from the conventional short axis view (Fig. 2). In both vessels, the Doppler sample volume was placed immediately distal to the semilunar valves. The Doppler sample volume length ranged between 0.1 and 0.3 $\mathrm{cm}$. Doppler tracings were accepted when the angle between the Doppler cursor and the assumed direction of flow was $15^{\circ}$ or less.

In each vessel the peak systolic velocity $(\mathrm{cm} / \mathrm{s})$ was determined and the ratio between both peak systolic velocities was calculated. Peak systolic velocities were measured from the zero-line to the highest point of the Doppler velocity peak. Blood flow velocity measurements in the umbilical artery were only performed in IUGR. In this vessel, attention was focused on the end-diastolic part of the flow velocity waveforms that was classified as present or absent. Doppler studies were performed by one examiner (I.A.L.G.) All flow velocity waveforms were recorded on hard copies. A microcomputer (Olivetti M24 Olivetti BV, Leiden, The Netherlands) linked to a graphics tablet was used for analysis of the Doppler recordings. The mean value over at least four consecutive flow velocity waveforms of optimal quality represented the peak systolic velocity in a particular pregnancy.

All Doppler studies were performed with the patient in a semirecumbent position and during periods of fetal apnea to avoid modulation of the waveforms by fetal breathing movements. Fetal heart rate ranged between 122 and $160 \mathrm{bpm}$ (mean $141 \mathrm{bpm}$ ). Statistical analysis included assessment of the relationship between peak systolic velocities in the cardiac outflow tract and gestational age by repeated measurements analysis of variance, for which the BMDP program 5V (BMDP statistical software manual, University Press of California, Berkeley, CA) was used. The 5 th and 95 th percentiles were constructed by taking the estimated regression line \pm 1.64 SD. The PSV measurements of the IUGR cases were expressed as SDS. The SDS of a value measured at a certain gestational age is defined as the distance expressed in SD of that value to the mean of the normal reference group at that gestational age.

\section{RESULTS}

In normal pregnancy $(n=46)$, acceptable flow velocity waveforms were obtained from the pulmonary artery in $147(80 \%)$ and from the ascending aorta in $139(76 \%)$ of the 184 Doppler

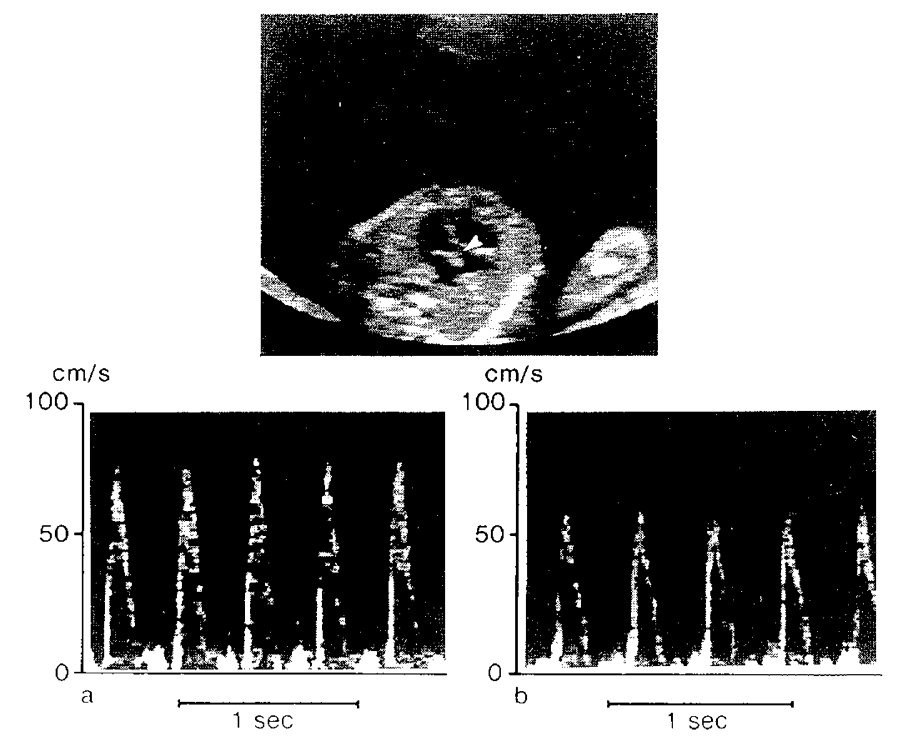

Fig. 1. Two-dimensional "five"-chamber view of the human fetal heart with Doppler flow velocity waveforms from the ascending aorta (arrow) during normal pregnancy $(a)$ and IUGR $(b)$.

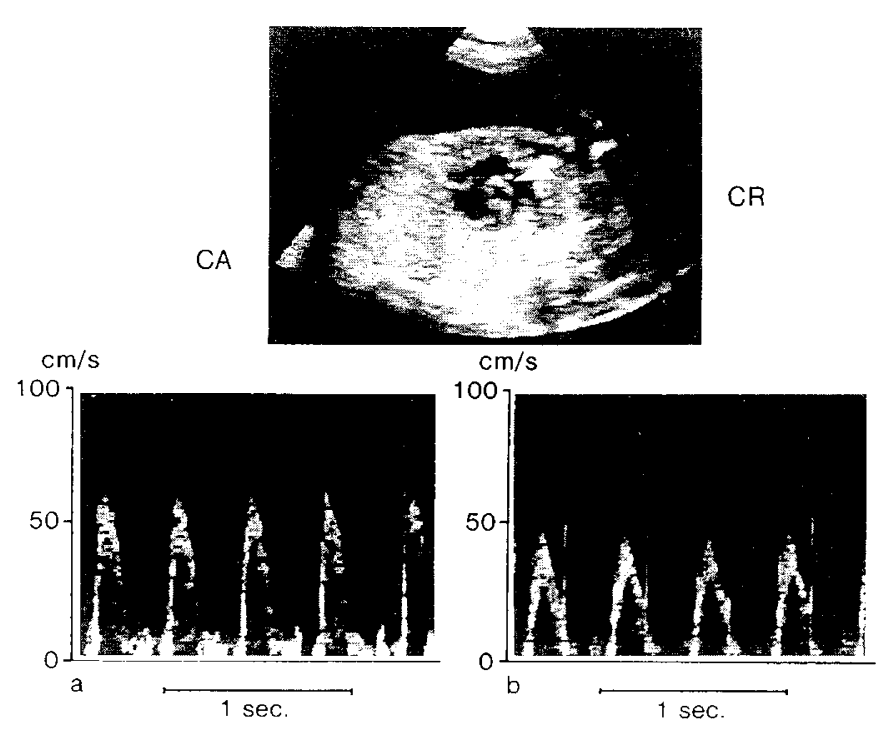

Fig. 2. Two-dimensional short axis/pulmonary arterial view of the human fetal heart with Doppler flow velocity waveforms from the pulmonary artery (arrow) during normal pregnancy $(a)$ and IUGR $(b)$. $C R$, cranial; $C A$, caudal.

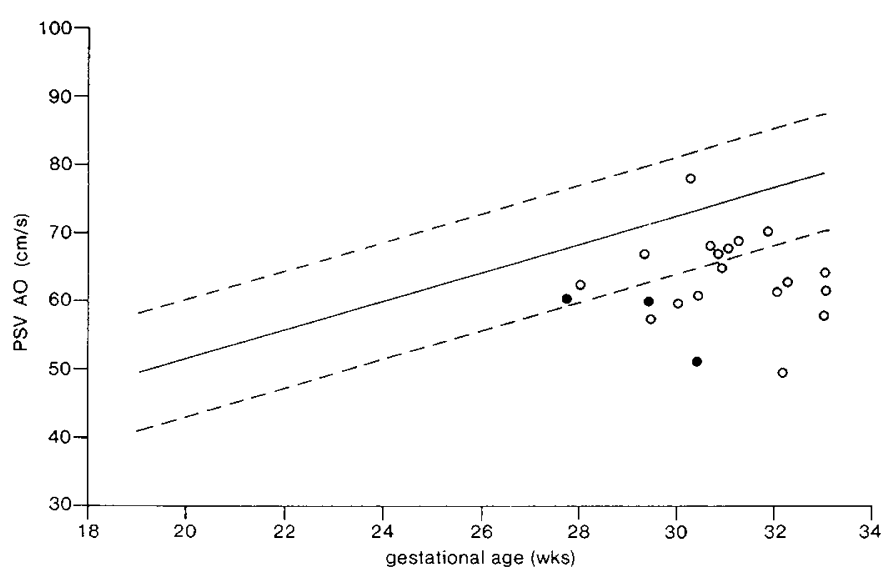

Fig. 3. Reference curves (5th and 95th percentile) for the PSV AO in normal subjects between 19 and $33 \mathrm{wk}$ of gestation, and data points from 21 IUGR cases. Regression equation for PSV AO $=9.21+2.12 \times$ age; $\mathrm{SD}=5.26$. Closed circles denote intrauterine deaths.

investigations. The success rate for obtaining acceptable waveforms from both arteries was $66 \%$. PSV in the ascending aorta and the pulmonary artery can be adequately described by a linear function of gestational age, with a constant residual SD around the regression line. The increase in PSV was statistically significant $(p<0.001)$ for both arteries. The mean PSV in the ascending aorta increased from $49.4 \mathrm{~cm} / \mathrm{s}$ at $19 \mathrm{wk}$ to $79.0 \mathrm{~cm} / \mathrm{s}$ at 33 wk. During the same gestational period the mean PSV in the pulmonary artery increased from 39.0 to $63.7 \mathrm{~cm} / \mathrm{s}$. The PSV ratio between the ascending aorta and pulmonary artery (PSV AO/PSV PA) remained virtually constant over the entire study period, with mean ratios ranging from 1.25 to 1.29 .

In Figures 3 and 4 the data points for the 21 cases of IUGR are shown with the reference curves. For the PSV in the pulmonary artery, $95 \%$ of IUGR cases had decreased ( $<5$ th percentile) values. For the PSV in the ascending aorta, $57 \%$ of IUGR cases had reduced ( $<5$ th percentile) measurements. In the umbilical artery, end-diastolic flow velocities were present in eight cases and absent in 13 cases. When comparing PSV values from the cardiac arteries to end-diastolic flow velocity in the umbilical artery, no significant relationship could be established. For the PSV from the ascending aorta the mean SDS (number of standard deviations from the mean) was -2.0 and -2.3 in the presence 


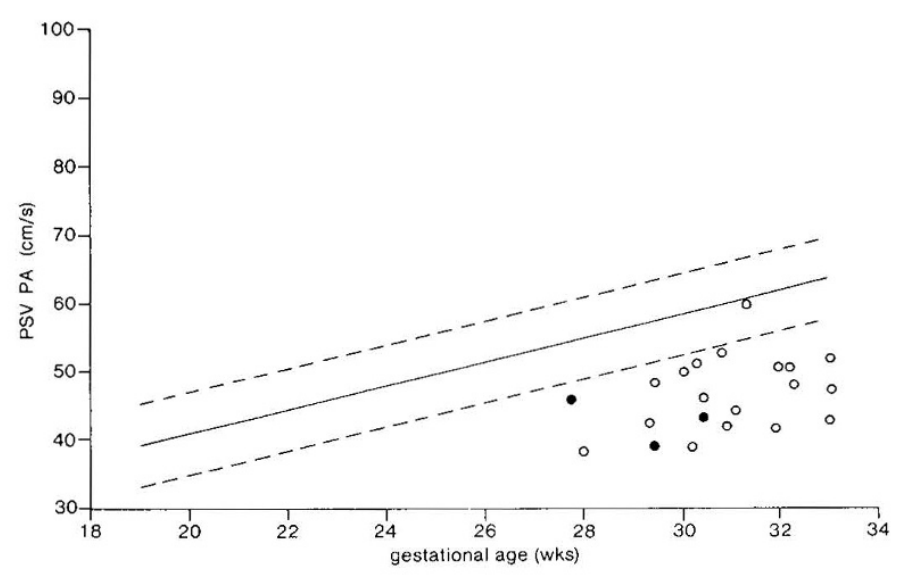

Fig. 4. Reference curves (5th and 95th percentile) for the PSV PA in normal subjects between 19 and $33 \mathrm{wk}$ of gestation, and data points from 21 IUGR cases. Regression equation for PSV PA $=5.48+1.77 \times$ age; $\mathrm{SD}=3.69$. Closed circles denote intrauterine deaths.

and absence of end-diastolic flow in the umbilical artery, respectively, whereas for the PSV from the pulmonary artery the corresponding scores were -3.2 and -4.1 . For the PSV from the ascending aorta, a statistically significant difference $(p<0.05$, Mann-Whitney test) in mean SDS was established when comparing birth weights below the 2.3 percentile $(-3.1)$ with birth wt between the 2.3 and 5 th percentile $(-1.7)$. No such difference could be documented for the PSV from the pulmonary artery, the SDS being -3.9 and -3.6 , respectively. No correlation could be established between fetal well-being, as reflected by the Apgar score at $1 \mathrm{~min}$ and umbilical arterial $\mathrm{pH}$, and the PSV in either artery. There were three intrauterine deaths that all occurred in utero within $7 \mathrm{~d}$ of delivery. Here, the PSV in either artery was not essentially different from those of the surviving IUGR cases.

\section{DISCUSSION}

This prospective longitudinal study describes the age-related changes in peak systolic flow velocities across the semilunar valves in the normal human fetus during the second half of gestation. The potential clinical use of flow velocity waveform recordings from the fetal ascending aorta and pulmonary artery is demonstrated by the higher than $75 \%$ success rate in obtaining acceptable recordings. Failure to obtain acceptable flow velocity waveforms from these vessels was usually due to excessive fetal movements or maternal obesity. The linear increase of peak systolic flow velocity in both arteries with advancing gestational age is in agreement with other studies $(2,5,7)$; our own absolute values are similar to those reported by Allan et al. (5), Huhta et al. (1), and Reed et al. (10), but lower compared with the studies by Hata et al. (2) and Kenny et al. (7). This may be due to the fact that angle correction that can give rise to velocity overestimation, was not applied in our study.

Cardiac performance is influenced by heart rate, preload, afterload, and intrinsic properties of both ventricles. From lamb studies it appears that cardiac function is particularly sensitive to changes in afterload (11), which is determined by blood pressure and vascular resistance. The gestational age-related rise in normal peak systolic flow velocity in the ascending aorta and pulmonary artery may be accounted for by increased volume flow through the semilunar valves, raised contractility, or reduced afterload. The noninvasive nature of the human fetal Doppler studies does not allow differentiation between these variables. However, age-related reduction in afterload may occur in the human fetus as a result of the physiologic decrease in placental vascular resistance as expressed by increased end-diastolic flow velocities in the umbilical artery during the second half of gestation (12). The relationship between peak systolic velocities in the ascending aorta and pulmonary artery appears to be constant during the 3rd trimester of pregnancy with PSV AO/ PSV PA ratio values of 1.25-1.29, indicating relatively higher peak systolic velocities in the ascending aorta. The latter could be explained by the fact that the afterload to the left ventricle may not only be determined by the umbilical placental and uteroplacental resistance but also by the relatively low resistance at the cerebral level $(13,14)$. Alternatively, the aortic valve size has been shown to be smaller than the pulmonary valve size (5) and as such may be responsible for the relatively higher peak systolic velocities in the ascending aorta.

The reduced peak systolic velocity values in the fetal ascending aorta and fetal pulmonary artery in IUGR due to impaired placental perfusion is in agreement with a previous cross-sectional study (15). Decreased peak systolic velocities could be secondary to reduced volume flow, increased semilunar valve or vessel size, reduced cardiac contractility, or increased afterload. Also, differentiation between these cardiac variables is impossible. However, the absence of tricuspid valve regurgitation makes impaired cardiac contractility less likely. Moreover, in a previous study on IUGR, aortic and pulmonary valve diameters were not essentially different from those observed in normal pregnancies (16). An increase in afterload to both ventricles may be expected on the basis of increased umbilical placental resistance as established by Doppler flow measurements (14). A considerable percentage $(43 \%)$ of the peak systolic velocities in the ascending aorta were within the normal range. We suggest that this may be determined by the marked reduction in cerebral vascular resistance in IUGR (14), which is in part responsible for the afterload to the left ventricle. This may also explain the relationship between PSV in the ascending aorta and birth wt percentile.

Fetal well-being as expressed by Apgar score and umbilical $\mathrm{pH}$ did not correlate with the peak systolic velocity in the fetal ascending aorta and pulmonary artery. We are aware of the fact that particularly the Apgar score is of limited significance in the assessment of fetal outcome.

It can be concluded that there is a linear increase of peak systolic velocity in the ascending aorta and the pulmonary artery during the second half of normal gestation. Higher peak systolic velocities were documented in the ascending aorta relative to the pulmonary artery. In both arteries reduced peak systolic velocities were observed during IUGR, which may reflect increased afterload to the cardiac ventricles. Whereas a positive correlation was established between the degree of disturbance in fetal growth and peak systolic velocity in the ascending aorta, this was not the case for the pulmonary artery. No correlation existed between peak systolic velocity in both arteries and Apgar score and umbilical cord $\mathrm{pH}$.

\section{REFERENCES}

1. Huhta JC, Strassburger JF, Carpenter RJ, Reiter A, Abinader, E 1985 Pulsed Doppler fetal echocardiography. J Clin Ultrasound 13:247-254

2. Hata T, Aoki S, Hata K, Kitao M 1987 Intracardiac blood flow velocity waveforms in normal fetuses in utero. Am J Cardiol 59:464-468

3. Reed KL, Sahn DJ, Scagnelli S, Anderson CF, Shenker L 1986 Doppler echocardiographic studies of diastolic function in the human fetal heart: changes during gestation. J Am Coll Cardiol 8:391-395

4. Maulik D, Nanda NC, Saini VD 1984 Fetal Doppler echocardiography: methods and characterization of normal and abnormal hemodynamics. Am J Cardiol 53:572-578

5. Allan LD, Chita SK, Al Ghazali W, Crawford DC, Tynan M 1987 Doppler echocardiographic evaluation of the normal human fetal heart. Br Heart $\mathbf{J}$ 57:528-533

6. De Smedt MCH, Visser GHA, Meyboom EJ 1987 Fetal cardiac output estimated by Doppler echocardiography during mid- and late gestation. Am J Cardiol 60:338-342

7. Kenny JF, Plappert T, Doubilet P, Saltzman DH, Cartier M, Zollars L, Leatherman GF, St John Sutton MG 1986 Changes in intracardiac blood flow velocities and right and left ventricular stroke volumes with gestational age in the normal fetus: a prospective Doppler echocardiographic study. Circulation 74:1208-1216

8. Kloosterman GJ 1970 On intrauterine growth. Int J Gynaecol Obstet 8:895912

9. Campbell S 1976 Fetal growth. In: Beard RW, Nathanielsz PW (eds) Fetal Physiology and Medicine. WB Saunders \& Co. Ltd. London, pp 271-279

10. Reed KL, Meijboom EJ, Sahn DJ, Scagnelli SA, Valdes-Cruz LM, Shenker L 
1986 Cardiac Doppler flow velocities in human fetuses. Circulation 73:4146

11. Gilbert RD 1982 Effects of afterload and baroreceptors on cardiac functions in fetal sheep. J Dev Physiol 4:299-309

12. Trudinger B, Giles WB, Cook CM 1985 Flow velocity waveforms in the maternal uteroplacental and fetal umbilical placental circulation. Am J Obstet Gynecol 152:155-163

13. Wladimiroff JW, Tonge HM, Stewart PA 1986 Doppler ultrasound assessmen of cerebral blood flow in the human fetus. Br J Obstet Gynaecol 93:471-475
14. Wladimiroff JW, Noordam MJ, Wijngaard van den JAGW, Hop WCJ 1988 Fetal internal carotid and umbilical artery blood flow velocity waveforms as a measure of fetal well-being in intrauterine growth retardation. Pediatr Res 24:609-612

15. Groenenberg IAL, Wladimiroff JW, Hop WCJ 1989 Fetal cardiac and peripheral arterial flow velocity wave forms in intrauterine growth retardation. Circulation 80:1711-1717

16. Reed KL Anderson CF, Schenker I 1987 Changes in intracardiac Doppler blood flow velocity in fetuses with absent umbilical artery diastolic flow. Am J Obstet Gynecol 157:774-779 